\title{
Infrared Radiometry Experiment for Mariner Mars $1971^{1}$
}

\author{
S. CHASE, JR. \\ Santa Barbara Research Center, Goleta, California 93017 \\ E. MINER \\ Jet Propulsion Laboratory, Pasadena, California 91103 \\ G. MÜNCH AND G. NEUGEBAUER \\ California Institute of Technology, Pasadena, California 91109
}

Received August 4, 1969 ; revised December 15, 1969

\begin{abstract}
The infrared radiometer is designed to provide brightness temperatures of the surface of Mars by measuring the energy radiated in the 8 to 12 and 18 to $25 \mu$ wavelength bands. The instrument is essentially the same as that flown on the Mariner Mars 1969 missions, modified only to define more sharply the field of view. Because Mariner Mars 1971 will orbit Mars, a given area of the planet will be observed at a variety of local times, and the characterization of the various areas by their thermophysical properties will be more complete than that obtained by Mariner Mars 1969.
\end{abstract}

The scientific objective of the infrared radiometry experiment on Mariner Mars 1971 is to measure, over a wide coverage of planetary surface, the temperatures of the soil as a function of local time. Information will be derived regarding: (1) thermophysical properties of the surface materials, (2) occurrence of irregularities in the cooling curve, other than those expected from albedo variations, and (3) existence of "hot spots," which may indicate sources of internal heat.

The rate of radiative cooling of an area on a planet with a thin atmosphere, and subject to insolation varying in time, depends on the parameter $(k \rho c)^{1 / 2}$, where $k$ is the thermal conductivity, $\rho$ is the density, and $c$ is the specific heat of the top surface layers. The actual functional dependence on local time of the temperature in the illuminated hemisphere depends

\footnotetext{
1 This work was performed for the Jet Propulsion Laboratory, California Institute of Technology, sponsored by the National Aeronautics and Space Administration, under Contract No. NAS 7-100.
}

primarily on albedo; in the night-time, it depends primarily on the value of $(k \rho c)^{1 / 2}$.

Because the infrared radiometer on Mariner Mars 1969 measured temperatures over a swath of planetary surface, the temperature, as a function of local time, could be established only in a mean sense. Significant variations in the thermophysical properties of the surface materials cannot be uniquely resolved from variations in albedo. In contradistinction, observations from Mariner Mars 1971 will provide cooling curves characteristic of individual areas; thus, the degree of correlation that may exist between characteristic cooling curves and major physiographic features can be found. There are extensive areas of the Moon that remain hotter than their surroundings at nighttime. Some of these irregularities are associated with the "rayed" craters, of probably recent origin. Because of the cratered nature of the Martian surface, it is natural to expect the existence of cooling anomalies in some Martian craters. Information regarding the aging process of 
various-sized craters will be derived from the Mariner Mars 1971 radiometer, whose spatial and thermal resolution will be comparable to that of Earth-based lunar measurements.

In function and design, the radiometer on Mariner Mars 1971 is essentially the same as that flown on Mariner Mars 1969 (Chase, 1969). However, after analysis of the data derived from the 1969 missions (Neugebauer et al., 1969), some minor modifications were made to the 1971 instrument. One undesirable feature of the 1969 instrument was its relatively high sensitivity to off-axis radiation, which was most readily shown by a nonzero response during the flyby, when the optical axes of both channels did not intercept, but were near, the planetary surface. By placing a focal-plane diaphragm in front of the bimetallic thermopile detectors, the fields of view of the Mariner Mars 1971 radiometer have been more sharply defined. The nominal size of the field of view of the 8 to $12 \mu$ channel has been reduced to $0.53^{\circ}$. The decrease in the net energy delivered to the detectors is inconsequential, as the performance of the Mariner Mars 1969 detectors actually surpassed that considered in the design.

The results of the infrared radiometry experiment on Mariner Mars 1969 were as anticipated in that the temperatures measured correspond closely to those of a model with mean thermophysical properties determined from Earth-based observations. The absence of pronounced thermal anomalies in the Mariner Mars 1969 data, however, must be judged in consideration of the very small fraction of the planetary surface observed (a total of about $1 \%$ ). The night-time picture coverage, in which thermal anomalies are most apparent, was only one-fifth of this percentage. It is believed that the wider coverage planned for 1971 (as much as $20 \%$ of the surface) will provide data to characterize accurately the thermophysical properties of the various physiographic features of the Martian surface and to establish the presence or absence of thermal anomalies in the planet.

\section{References}

Chase, S. C., JR. (1969). Infrared radiometer for the 1969 Mariner mission to Mars. Appl. Optics 8, 639 .

Neugebauer, G., Münch, G., Chase, S. C., Jr., Hatzenbeler, H., MiNer, E., AND Schofield, D. (1969). Mariner 1969: Preliminary results of the infrared radiometer experiment. Science 166, 98-99. 